\title{
OPERATIONAL EXPERIENCE WITH MODEL-BASED STEERING IN THE SLC LINAC*
}

\author{
K. A. THOMPSON, T. HIMEL, S. MOORE, L. SANCHEZ-CHOPITEA, ANO H. SHOAEE \\ Stanford Linear Accelerator Center \\ Stanford linjersity. Stanford, California 94309
}

\section{ABSTRACT}

Operational experience with model-driven steering in the linac of the Stanford Linear Collider is discussed. Important issues include two-beam steering, sensitivity of Algorithms to faulty components, sources of disagreement witb the model. and the effects of the finite resolution of beam position moni. tors. Methods developed to make the steering algorithms more robust in the presence of such complications are also prestented.

\section{INTRODUCTION}

In the $3 \mathrm{~km}$ linac of the Stanford Linear Collider, it is necessary to keep the beam wilhin about $100 \mu \mathrm{m}$ of the axis of the accelerating structure in order to avoid emittance growth due to the transwerse wake field. In this paper, we discuss the steering algorithms that bave been found most usefol in the linac. with emphasis on operational experience in the normal mode of SLC operation in which $e^{+}$and $e^{-}$bunches of bigb intensity (at present up to about $3 \times 10^{10}$ particles per bunch) must be steered simultapeously. More general discussions of the SLC steering ajgorithms and soltware implementation appear elsewhere. ${ }^{\text {(I) }}$ The lianc consists of $100 \mathrm{~m}$ sectors, exch containing eight girders; at the end of each girder of a typical sector is one quadrupole magnet of the FODO lattice. The first sector after the damping rings has four times as many quads, and the next two seclors each have twice as many, in order to provide proportionately smaller beta functions where the low-energy beams from the damping tings are more sensitive to wakefields. The phase advance per cell is $90^{\circ}$ in ahout the first half of the linac, then tapers to about $45^{\circ}$ at the end of the linac as the quads saturate. Installed in the bore of each quad is a stripline beam position monitor (BPM). Each BPM is gated so that it can messure the position of either an $e^{+}$or $e^{-}$bunch on given machine pulse. A short distapce after exch quad is a pair of dipole corrector magnets, for steering in the horizontal (X) and vertical (Y) directions.

\section{STEERTIG ALGORTTHMS}

\subsection{The Basic 1-to-1 Steering Algorithm}

The basic steering algorithm in the SLC linac utilizes a oneto-one matehing between correctors and downstream BPMs, for each direction $(X$ or $Y)$ and beam $\left(e^{+}\right.$or $\left.e^{-}\right)$. For each beam and trapsverse direction, the one-to-one steering algoritbm uses a cortector jubl afler a focusing quadrupole to zero the BPM reading in the next downstream focusing quadrupole. Thus each beam is being corrected at the BPMs where its beta function is larges, and is left to fend for itself at the BPMs in the defocusing quadr, where its beta function is smallest. In this way $e^{+}$und $e^{-}$beams are simultaneously steered.

The general setup is shown in Fig. 1 for the case of one of the beams. The correctors not used for this beam are used for the other beam in a complementary manner (sot the discussion of two-beam steering in Sec, 2,3, below').

One may correct the orbit in either or both of the $X$ and $Y$ directions at a time. The kick(s) are calculated to "zero,"

Work supported by the Department of Energy, contract DE-AC03-76SF00515.

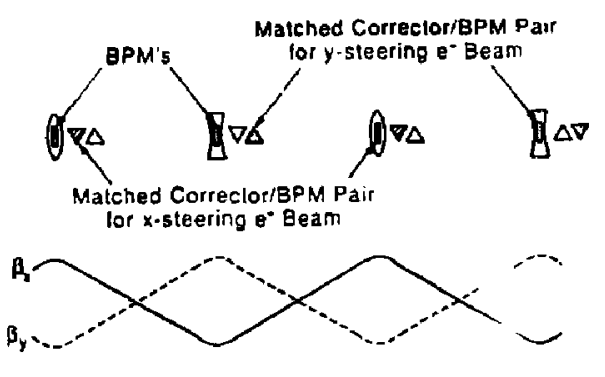

0 Quad loeusing $\theta^{-}$beam in $\times$direction

$\rrbracket$ auad locusing $e^{-}$beam in y direction

$\nabla \times$ corrector $\Delta y$ corrector $\square$ BPM

Fig. 7 . The matching between correctors for one of th. two beams, say the electran bedm. Note that the matched $c$ reclor/BPA pairs are nearest the focusing quads for the dir ion ( $X$ or $Y$ ) being steered. The remaining correctors are $w$ fo steer the other beam.

the reading on the matched BPM in the desited direction(s), then the efiect at al] downstream BPMs is calculated. (Note that one may actually steer wo specified desired ofisets in BPMIs rather than literally zero, but in our discussion we shall simply refer to this as zeroing the BPMs.) In steering a given region of the linac, the algorithm proceeds downstream through the matched corrector/BPM sets in the region. In general, one ends up with smallest offsets of the beam at the matched BPMIs, and larger (but still acceptable) offets at the unmalched ones.

\subsection{The Robust, 1-to-1 Steering Algorithm}

It is often the case that one or more BPMs or correctors in a region are broken. Note that the simple 1-to-1 ajgorithm makes no change to a corrector whose metched BPM is broken. and makes no attempt to zero a BPM whose matched corrector is brokea. A new "robust" algorithm was developed that steers on a olightly more global level when something is broken. in order to overcome these limitatjons. The basic idea is to work with a group of BPMs and correctors in a region containing one or more broken components. If there are more BPMs than correctors in the group, the RMS of the BPM readings is minimized, and if there are more correctors than BPMs, the RMS of the corrector strengths is minimized. Details of the algorithm are given in the Appendix.

\subsection{Two-Beam Steering}

For a given beam, $\beta_{z}$ and $\beta_{y}$ are out of phase, and for a given direction ( $X$ or $Y$ ) the beta function is out of phase be tween the two beams. The beta functions reach their maxima and minima at alternate quads, with the ratio $\beta_{\text {mar }} / \partial_{\min } \approx 4$. Thus, as noted above, one may steer one of the two beams without baving a large effect on the other one. There is some residual eflect however, so what is done is to iterate back and forth 
Wiween the two beams. Typically, the orbit of the positron beam has somewhat larger RMIS offsets than the orbit of the electron beam, so the best results are obtained by starting with the positron beam. Lodeed, if one starts by steering the electron beam when the positron orbit is relatively bad, one can end up losing the positron beam completely.

The overall two-beam steeriog ajgorithm is as tollows:

1. Calculate the settings of the correctors for the position bean that should 2ero its orbit at the matched positron BPMs.

2. Predict the change in the orbit of the electson beam due to these new positron corrector settings.

3. Calculate the settings of the correctors for the electron bean that should zero its orbit at the matched electron BPMs.

4. Predict the resulting change in the orbit of the positron beam.

5. Continue iterating steps I through 4 , correcting the pre dicted orbit of one beam and calculating the effect on the other bearn. Simulations and experience with the actual machine have led to an implementation having a total of three such iterations.

6. Trim the correctors in the linac to the final calculated settings.

Note that we are in effect inverting a matrix by iteration. This is much foster than a direct inversion since the matrix may 1 e guite large $(300 \times 300)$.

\section{PHASE ADVANCE IN MODEL AND ACTUAL MACHINE}

As noted above, an essential part of the steering aigoritbms is being able to predict the downstream effects of a change in corrector setting. In particular, the betatros phase advance predicted by the machine model needs to agree witb the actual phase advance over the region of the limac being steered. There are about 30 betatron osciltations in the length of the linac, and one would like to have the actual and predicted phase advance agree over the entire length, since sny significant discrepancy makes it necessary to steer in sborter segments over wbich the model and machine phase advance remain fairly coberent. For this and otber reasons, metbods have been developed to characterize the phase advance, diagnose and fix the causes of discrepancies where possible, and compensate for the effects of any discrepancies whose origins remain uncertain." The basic jdea of this "lattice diagnostic" program is to introduce a betatron oscillation in the actual machine, compare the phase advance with that predicted by the model, then vary the model energy profle to obtain the best possible agreement between the predicted and actual phase advance. The program outputs the apparent energy erron required to obtain the fit. Obviously, large phase advance errors should be fixed at their source where possible. However, to take account of any remaining model/machine disagreement, "Fudge factors" can be computed from che apparent evergy exrors, to enable more efficient steering.

\section{BEAM POSITION MEASUREMENT EFEECTS}

Tbe beam position monitors measure the transverse poaition of bunch centroids. The bunches bave a transverse extent comparable to the offsets one is interested in (ol order $100 \mu$ ), and a length of approximately $1 \mathrm{~mm}$. Wakefield-induced tails are a potential problem for steering. since they enlarge and distort the bunch. By using BXS damping:" such tails hase. been successfully controlled in the SLC linac at intensities up to about $3 \times 10^{\text {id }}$ particles per bunch". and the steering algu. rithms bave continued to work under these conditions. We hav. yet to obtain experience witb bigber currents, but it is experted that use of BNS danping will allow sufficient control of wah" field effects in this regime as well.

Ideally, after enough iterations of the robust. One-to-one stering algorithm to eliminate residual coupling offer s. one would end up with all good, matched BPMs zeroed. There are, of course, limits in reality, due to the finite resolution of the BPMs and to beam jitter. It is possible, at present. to surcess[ully stere botb beams to about the desised 100 , RMS offsets in both transverse directions. There is still room for improvement in the speed with which this can done. whirh will come about as it becomes possible to predict the orbits more accurately over longer regions.

\section{OTHER PLANNED IMPROVEMENTS AND DXTENSIONS}

The present steering software is based on the Twiss parant eter representation of the machine model for the horizontal and vertical directions, and thus is not suited for handling regions where there is $X / Y$ coupling. Steering in the linar is essen. tially uncoupled in $X$ and $Y$, but this is not the case in some other parts of the SLC, fo: instance the arcs fiom the end of the linac to the fiual focus. Thus, tbere has been a tendenty to bandle steering in different parts of the SLC in a rather disjoint mannet. Work is currently in progress to base the steering software throughout the SLC on the fully-coupled transfer matrices rather than Twiss parameters. It is hoped that this will allow more general algorithms and more continuity in sleering the beam through larger regions of the SLC

\section{ACKNOWLEDGMENTS}

We would like to acknowledge the work of 1 . Almog. who wrote much of the original stcering code. We thank $j$. Steman, C. Adolptsen. M. Woodley, and the SLC operations staft for useful discussions. feedback, and help in commissioning the* systems discussed bere.

\section{APPENDIX}

For simplicity: we describe the robust oneto-one steering algorithm for the case of steering one of the two beams in one direction, say electrons in the $X$ direction. Denute the transfer matrix element from as angle at a corrector to the resulting offet at a BPM by $T_{12}$. Thus, we initially have nalched pairs of X correctors and BPMs, and associated with each correclor in a pair there is a transfer matrix element $T_{12}^{\text {mateh }}$ to its matched BPM. However, some of the correctors or BPMs may be bad. so we do the following, proceeding downstream from the first noatched pair:

1. If the BPM/corrector pair is to be used for positrons onlygo to the next matched pair.

2. Else if both the BPM and corrector are bad, then go on to the next pair.

3. Else if botb the BPM and corrector are good, see if there is a bad corrector in either of the next two dow rstrean! matched pairs for electrons.

(a) If there is such a bad corrector, and $T_{12}$ from the present corrector to the BPM matched to the bad corrector is at least 0.3 of the $T_{12}^{\text {mateh }}$ of the bad corrector, then all the electron correctors from the present corrector, up to but not including the bad one, will be used to minimize the RMS of the BPMs matched to those correctors plus the one matched to the bad corrector.

s.

.

.


(b) Otherwise, the present good corrector will be used to zero the present good BPM as in the simple one-toope algorithm.

4. Else if the corrector is bad and the BPM is good, go on to the next matched pair. Sote that according to the previous slep. we used a previous correctot to minimize the reading of the BPM matched to this bad corrector, if it ras possible.

5. Else if the cofrector is good but the BPM is bad. then look for other BPMs to help determine the setting of this conector, as follaws:

(a) First whether all of the following hoid:

(i) The matched BPMs immediately before and after the present BPM are intended for use only on the other beam, Damely positrons.

(ii) These two BPAls are both good, and they bave $T_{1}{ }^{\prime}$ 's to the present corrector of at least $0.2 T_{12}$ afch.

(iii) The next BPM designated for electrons only is good. Then these three good BPMs, the present correstor, and the corrector matched to the third BPM are grouped together. We then zero the reading of the third BPM and minimize the RMS of the other two. (b) Otkerwise. look downstrean for a goud elertron BPM with a $T_{12}$ from the present corrector of at leas: $0.3 T_{12}^{\text {math }}$, If we come 10 a bad electron BPII at a bad electron corrector before finding such a BPYW or if we don't find such a BPM within the next is BPMs, or if we find such a BPAl but its matrind corrector is bad, then give up and ga on to the next BPM/cotrector pait. If we do find such \& BPMI. thr'It Al electron BPMs and correctors betweth the pueneth pair and that BPM are grouped together. Since thert should be one morc corrector than BPM in the grous. we zero the BPMs and minimize the RMS of the cor rector kicks.

\section{REFERENCES}

1. J. Sheppard el ol., "Beam Steering in the SLC Linar. "IEEE Particle Ascelerator Conf., Vancouver (1985)

2. 1. Almog. H. Shoaee ef al., in preparation.

3. T. Himel and K. Thompson, Energy Measutements from Betatron Oscillations," these Proceediags.

4. V. Balakin. A. Novakhatsky and V. Smirnov, Pror of the t2th Int. Conf. on Hagh Encrgy Accelerators. Feruilah (1983).

5. J. T. Seeman et al. "Transverse Wakefield Damping (BNS-Landau Damping) in the SLC Linac." these Proceedings.

\title{
DISCLAIMER
}

\begin{abstract}
This report was prepared as an account of work sponsored by an agercy of the Uniled Stazes Government. Neither the United States Government nof any agency thureof, nor any of their employtes, makes any warranty, express or implied, of assumes any legal liability or tesponsibility for the accuracy. completeness, or usefulness of any information. apparatus, product, or process disclosed, or represenls that its use would not infringe privately owned rights. Reference herein to any specific commercial product, process, or service by rade name. trademark. manufacturer, or otherwise does nol necessarily constitute or imply its endorsement, recommendation, or faworing by the Unilod States Government or any agency thereof The views and opinions of authors expressed herein do not necessarily state or reflect those of the Lnited States Goversment or any agency thercof.
\end{abstract}

